\title{
Nuevos datos sobre Andegameryx (Mammalia, Ruminantia) del Mioceno Inferior de las Bardenas Reales (Navarra, España)
}

\author{
New data on Andegameryx (Mammalia, Ruminantia) from the Lower \\ Miocene of Bardenas Reales (Navarre, Spain)
}

\author{
V. Quiralte ${ }^{1}$, X. Murelaga², J.C. Larrasoaña ${ }^{3}$, H. Astibia² ${ }^{2}$ J. Morales ${ }^{1}$
}

\begin{abstract}
RESUMEN
En este trabajo se describen los nuevos hallazgos de rumiantes fósiles del Mioceno inferior (Rambliense, MN3) de las Bardenas Reales (Navarra). Este material está compuesto por dentición y ha sido clasificado como Andegameryx aff. andegaviensis Ginsburg, 1971. El estudio morfológico y métrico muestra que los restos de las Bardenas son muy semejantes a los de la población española de Andegameryx andegaviensis de Cetina de Aragón (Zaragoza), de talla algo mayor que las poblaciones francesas de esta especie. Sin embargo, también tiene afinidades con material de Andegameryx procedente de otras localidades españolas como Loranca del Campo (Cuenca). Los nuevos hallazgos permiten confirmar sin dudas la presencia de Andegameryx en las Bardenas Reales (Cuenca del Ebro), poniendo de manifiesto que este género de rumiante estuvo ampliamente extendido en las cuencas interiores de España durante el Mioceno inferior.
\end{abstract}

Palabras clave: Andegameryx, Mioceno inferior, Rambliense, Bardenas Reales, Navarra

\begin{abstract}
New fossil remains of ruminants from the Lower Miocene (Ramblian, MN3) of the Bardenas Reales (Navarre) are studied in this paper. The material, which comprises dentition, is assigned to Andegameryx aff. andegaviensis Ginsburg, 1971. Morphological and metrical similarities exist between these remains and the Spanish population of Andegameryx andegaviensis from Cetina de Aragón (Zaragoza), which is somewhat larger than the French material of this species. However, there are also some affinities with Andegameryx fossils from other Spanish localities as Loranca del Campo (Cuenca). The new material from the Bardenas Reales allows us to confirm certainly the presence of Andegameryx in the area and to provide the evidence that it was a widespread genus of ruminant in Spain during the early Miocene.
\end{abstract}

Key words: Andegameryx, Lower Miocene, Ramblian, Bardenas Reales, Navarre

\section{Introducción}

Los potentes depósitos del Mioceno continental de las Bardenas Reales de Navarra y áreas colindantes (Cuenca del Ebro) contienen asociaciones fósiles de vertebrados que abarcan un lapso temporal comprendido entre el Ageniense y Aragoniense (MN2 a MN4/5, Mioceno inferior-medio) (Murelaga, 2000; Murelaga et al., 2004a; Ruiz-Sánchez et $a l$., en prensa a, en prensa b). A excepción de Cainotheriidae, los fósiles de Artiodactyla son muy escasos en la zona. Hasta la fecha, los únicos restos

\footnotetext{
1 Museo Nacional de Ciencias Naturales, Departamento de Paleobiología, José Gutiérrez Abascal, 2.28006 Madrid.

Email: vickyq@mncn.csic.es, jorge.morales@mncn.csic.es

2 Universidad del País Vasco/Euskal Herriko Unibertsitatea, Facultad de Ciencia y Tecnología, Departamento de Estratigrafía y Pale-

ontología, Apartado de correos 644.48080 Bilbao. Email: xabier.murelaga@ehu.es, humberto.astibia@ehu.es

3 Instituto Geológico y Minero de España, Unidad de Zaragoza, Manuel Lasala 44, 9B. 50006 Zaragoza. Email: jc.larra@igme.es
} 


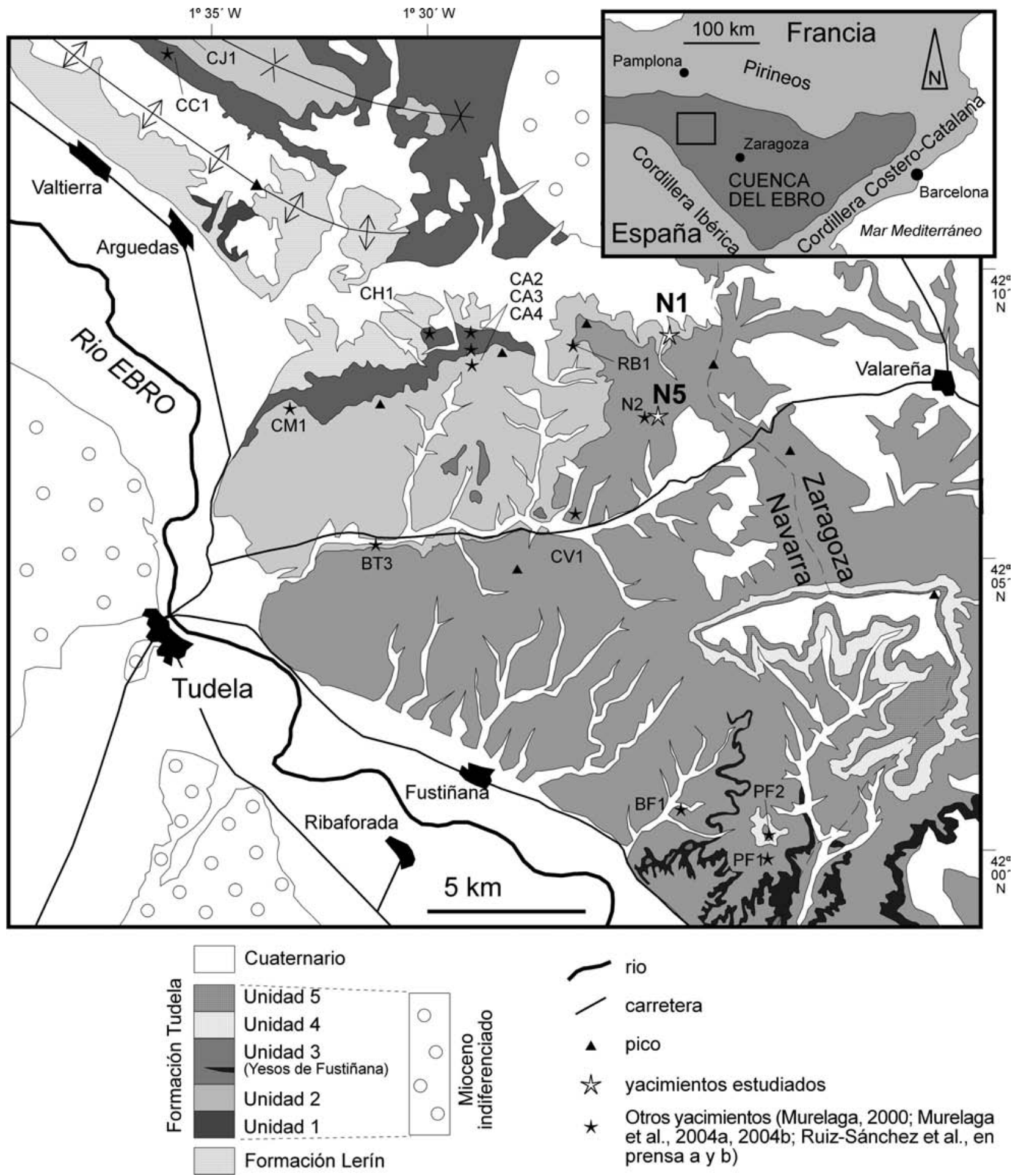

Fig. 1.-Mapa geológico del entorno de las Bardenas Reales de Navarra, en el que se han situado los yacimientos objeto de este estudio (Nasa 1 y Nasa 5), así como otros yacimientos de la Formación Tudela estudiados en trabajos previos. 
de rumiantes Pecora conocidos eran elementos postcraneales asignados a los géneros Teruelia, Procervulus? y Andegameryx (Murelaga et al., 2004a). El estudio de la dentición hallada en los yacimientos de Nasa 1 y Nasa 5 que presentamos a continuación permitirá un mayor conocimiento de esta forma de Andegameryx.

\section{Marco Geológico}

Las Bardenas Reales de Navarra se asientan sobre los materiales continentales Miocenos de la Formación Tudela (fig. 1). Dicha formación se apoya concordante sobre los yesos de la Formación Lerín, y sus tramos superiores constituyen los materiales más recientes conservados en el sector navarro de la cuenca terciaria del Ebro. La Formación Tudela está constituida por lutitas de tonalidades rojizas, ocres, amarillentas y grisáceas, entre las que se intercalan niveles de orden centimétrico a métrico de calizas, areniscas y yesos. Estos materiales se depositaron en la zona de transición situada entre los lagos que ocupaban el sector central de la Cuenca del Ebro, que en ocasiones tenían carácter evaporítico, y las extensas llanuras aluviales distales que drenaban la Cordillera Ibérica y los Pirineos (Alonso-Zarza et al., 2002; Larrasoaña et al., 2006). La Formación Tudela contiene numerosos yacimientos de vertebrados fósiles (Murelaga, 2000), que incluyen una variada fauna de micro- y macro-mamíferos (Murelaga et al., 2004a, 2004b; Ruiz-Sánchez et al., en prensa a, en prensa b), reptiles y anfibios (Murelaga et al., 2002) y aves (Murelaga, 2000). El contenido en mamíferos permite asignar dichas faunas a las biozonas locales que van de la $\mathrm{Y}$ a la $\mathrm{C} / \mathrm{D}$ (biozonas MN2 a MN4/5; Daams et al., 1987). El estudio magnetoestratigráfico llevado a cabo en los materiales de la Formación Tudela (Larrasoaña et al., 2006) ha permitido acotar su edad, de acuerdo con la ATNTS2004 (Lourens et al., 2004), entre los 20.2 y 15.5 Ma. En la parte media de la Formación Tudela destacan los yacimientos de Nasa 1 y Nasa 5 (fig 1; fig. 2), que son objeto de estudio en este trabajo. Dichos yacimientos se corresponden con niveles lutíticos de color gris, acumulados en medios palustres, y se asignan a la biozona local A (MN3) según su contenido paleontológico (Murelaga, 2000; Murelaga et al., 2004a). La correlación litoestratigráfica entre los yacimientos Nasa 1 y Nasa 5 y las series estratigráficas vecinas donde se han obtenido datos pale-

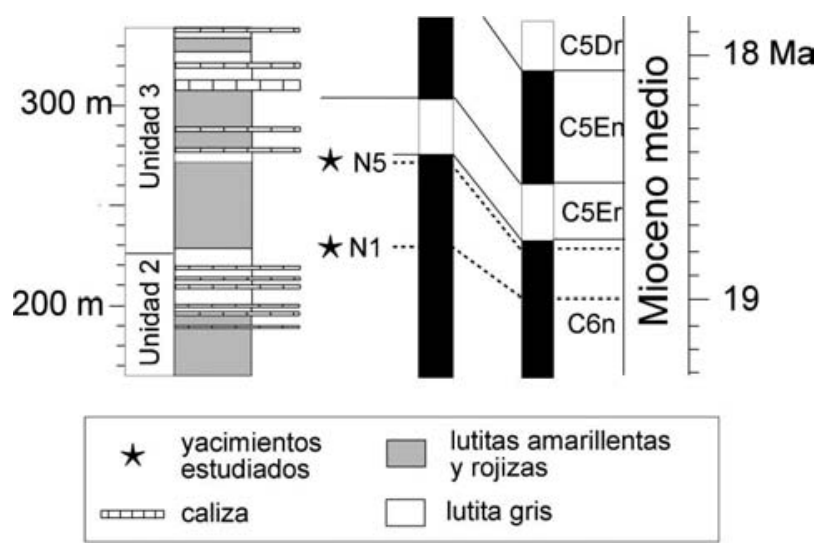

Fig. 2.-Situación de los yacimientos Nasa 1 y Nasa 5 en la columna compuesta de la Formación Tudela (Larrasoaña et al., 2006) y su correlación con la ATNTS de Lourens et al. (2004).

omagnéticos, permiten situar dichos yacimientos en la parte alta del cron C6n (Larrasoaña et al., 2006), con una edad aproximada de 19 y $18.8 \mathrm{Ma}$., respectivamente (fig. 2).

\section{Paleontología Sistemática}

Orden Artiodactyla Owen, 1848

Suborden Ruminantia Scopoli, 1777

Infraorden Pecora Linnaeus, 1758

Familia Andegamerycidae Ginsburg \& Morales, 1989

Género Andegameryx Ginsburg, 1971

Andegameryx aff. andegaviensis Ginsburg, 1971

\section{Material}

N1-283: fragmento de hemimandíbula izquierda con m1-m2. N5-65: fragmento de $\mathrm{m} 3$ derecho.

Localidades: Nasa 1 (N1) y Nasa 5 (N5), Bardenas Reales, Navarra

Edad: Rambliense (Mioceno inferior)

\section{Descripción}

En N1-283 se conserva parte del hueso mandibular junto al m1 y m2, que están completos y bien conservados (fig. 3: a y b). Los molares son piezas relativamente braquiodontas y selenodontas. El $\mathrm{m} 1$ tiene un desgaste medio y el $\mathrm{m} 2$ está poco desgastado. En ambos molares la muralla de los cónidos linguales está algo abombada. Las crístidas y cónidos linguales se encuentran más alineados entre sí en el m1 que en el m2. En el m1 puede verse cómo las crístidas posteriores del metacónido y protocónido se unen con la preentocrístida al avanzar el desgaste. La postentocrístida está desarrollada, aunque no llega a juntarse posteriormente con la posthipocrístida, por lo que el extremo postero-lingual del diente está abierto (fig. 3: a y c). 


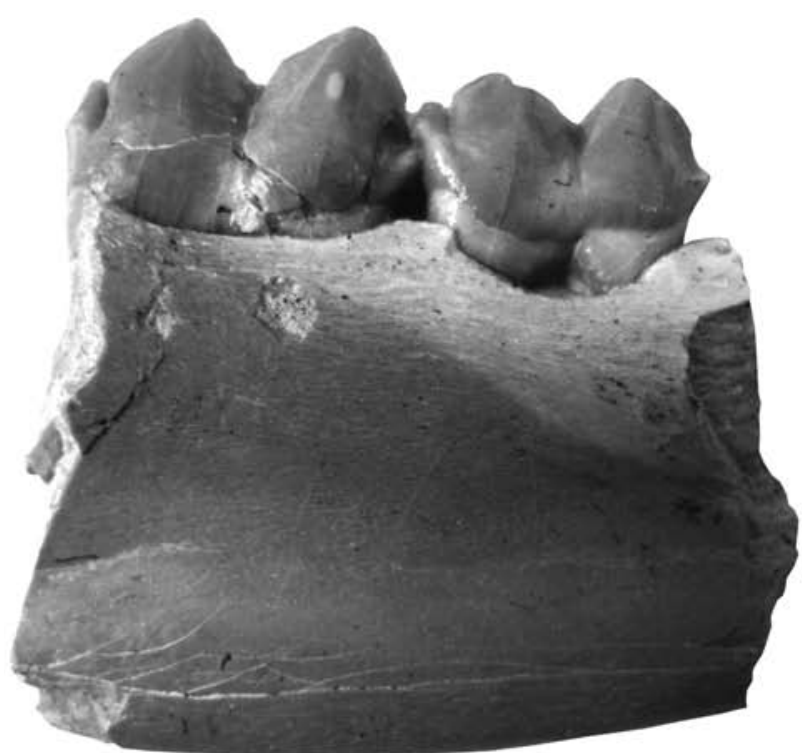

a

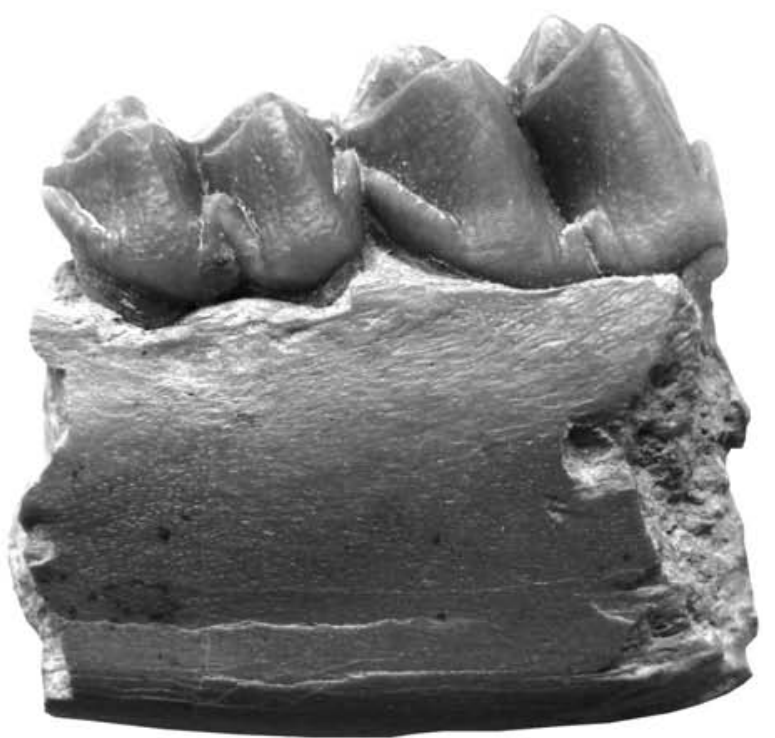

b

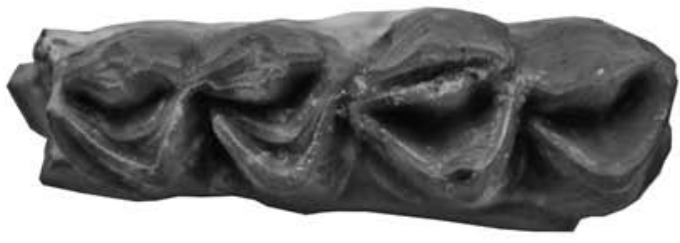

c

\section{$1 \mathrm{~cm}$}
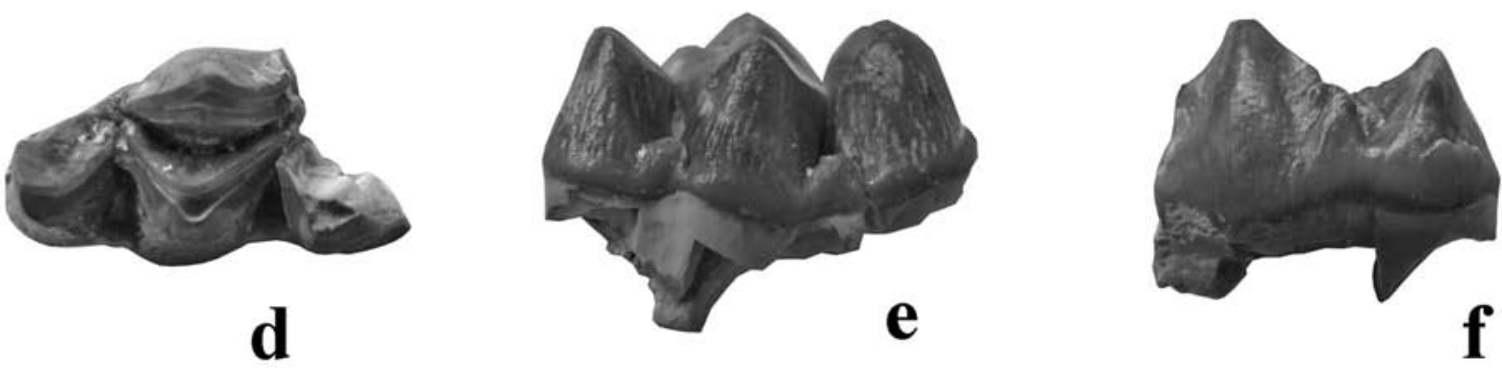

Fig. 3.-Andegameryx aff. andegaviensis de los yacimientos de Nasa 1 y Nasa 5, Bardenas Reales (Navarra). a, b, c. N1-283 fragmento de hemimandíbula izquierda con $\mathrm{m} 1-\mathrm{m} 2$. a) vista lingual, b) vista labial, c) vista oclusal. d, e, f. N5-65 fragmento de m3 derecho. d) vista oclusal, e) vista labial, c) vista lingual.

No se observa metastílido en el m1 y tampoco parece existir en el $\mathrm{m} 2$, si bien en este molar existe una pequeña muesca en el esmalte de esta zona. Sin embargo, cabe destacar la presencia de restos de un débil pliegue paleomerícido en ambos molares (fig. 3: c). En vista labial puede verse que los ectostílidos son fuertes (fig. 3: b). En ambos molares el cingúlido labial anterior es muy fuerte, observándose en el m1 pliegue caprino (fig. 3: c).

El m3 N5-65 está incompleto, ya que le falta el metacónido. El grado de desgaste es medio. La muralla lingual del entocónido está algo abombada y la postentocrístida, aunque desarrollada, no llega a cerrar por completo el diente en su ángulo postero-lingual (fig. 3: d). En vista labial se aprecian dos ectostílidos fuertes (fig. 3: e). El tercer lóbulo es sencillo y está formado sólo por un hipoconúlido alto y picudo que tiene un cingúlido en su base (fig. 3: f).

\section{Medidas}

\begin{tabular}{lccc}
\hline Sigla & Elemento & Longitud & Anchura \\
\hline N1-283 & $\mathrm{m} 1$ & 11,4 & 7,1 \\
& $\mathrm{~m} 2$ & 12,3 & 8,1 \\
\hline N5-65 & $\mathrm{m} 3$ & $19,5 *$ & 8,6 \\
\hline
\end{tabular}

El asterisco indica medidas aproximadas. Medidas tomadas en $\mathrm{mm}$. 


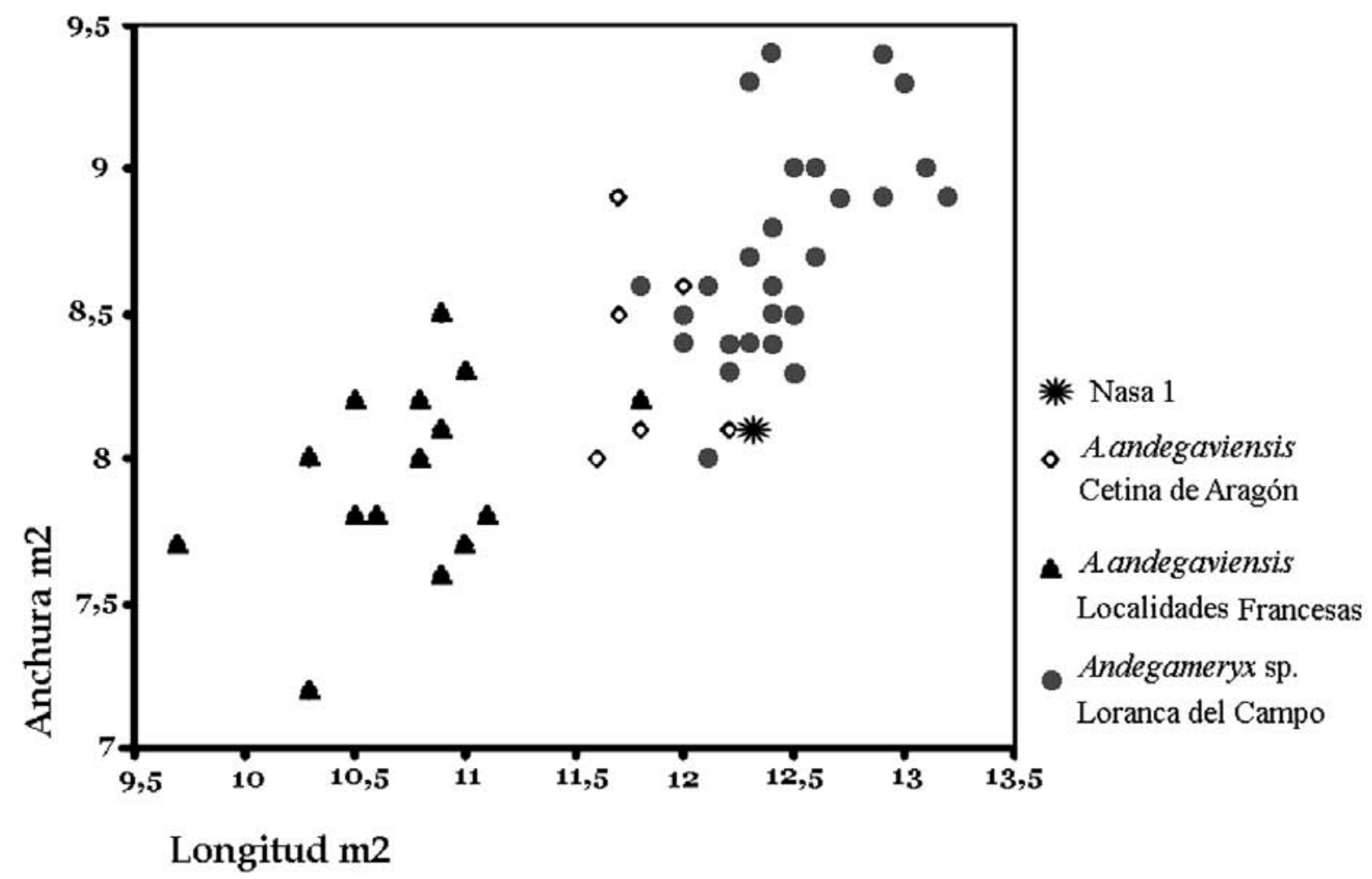

Fig. 4.-Gráfico de dispersión de la longitud y anchura del m2 de varias especies de Andegameryx, procedentes de localidades del Mioceno inferior (MN2-MN3) de Francia y España (Cetina de Aragón y Loranca del Campo). Las localidades francesas incluyen Chavaignes, Pont-Boutard, Les Beilleaux, La Brosse, Chitenay y Pontigné (localidad tipo del género). Todos los datos proceden de observaciones propias de los autores.

\section{Discusión}

La presencia de Andegameryx en yacimientos del Mioceno inferior de las Bardenas Reales de Navarra ya fue señalada por Murelaga et al. (2004a) a partir de material postcraneal procedente de la localidad de Barranco de Tudela 3 (ver fig.1). En aquel momento esta forma fue clasificada como Andegameryx sp. Los nuevos hallazgos de las localidades de Nasa 1 y Nasa 5 incluyen dentición, lo que nos ha permitido determinar los restos de manera más precisa.

Los molares inferiores son bastante sencillos, relativamente bunodontos, sin metastílido, con un débil pliegue paleomerícido y el tercer lóbulo del $\mathrm{m} 3$ simple. Este conjunto de caracteres morfológicos es propio del género Andegameryx, establecido por Ginsburg en 1971 a partir de material de la localidad francesa de Pontigné (zona MN3, Mioceno inferior). En concreto, la especie tipo del género, A. andegaviensis, presenta los mismos rasgos morfológicos generales que los descritos en los restos de las loca- lidades de Nasa 1 y Nasa 5. Sin embargo, hemos comparado dicho material con los datos disponibles de A. andegaviensis de varias localidades francesas y de la localidad española de Cetina de Aragón (biozona Y, MN2; Ginsburg et al., 1994), encontrando algunas diferencias en la morfología y en la talla. Así, los molares de Nasa 1 y Nasa 5 son proporcionalmente más estrechos, más comprimidos, que los molares de A. andegaviensis (fig. 4). Las piezas son también algo menos bunodontas y más selenodontas que las homólogas de A. andegaviensis. Tenemos que señalar que, si bien se trata sólo de tres ejemplares, los restos estudiados carecen de metastílido, que es un elemento reducido, pero que puede estar presente con cierta frecuencia en A. andegaviensis. En cuanto a las dimensiones, la dentición de Nasa 1 se encuentra en el rango de variación de la población de A. andegaviensis de Cetina de Aragón, que es, en general, algo mayor que las poblaciones francesas de esta especie (fig. 4).

Por tanto, aunque la morfología general de los restos de dentición de Nasa 1 y Nasa 5 es bastante 
semejante a la de A. andegaviensis, hemos encontrado algunas diferencias morfológicas y de talla. Estas diferencias son comparables a las que existen en algunas formas presentes en otros yacimientos españoles del Mioceno inferior (Loranca del Campo, Ramblar, Ágreda, La Encinilla), que tradicionalmente han sido incluidas en el género Andegameryx (Morales et al., 1986, 1999; Quiralte \& Morales, 2006; DeMiguel et al., en prensa). A este respecto, hemos de comentar que el género Andegameryx está siendo revisado actualmente por uno de los autores (V. Q. Tesis Doctoral) y que en un futuro cercano veremos ampliado el conocimiento de las formas presentes en estos yacimientos españoles, incluyendo además las localidades de las Bardenas Reales. Por tanto, en espera de completar dicho estudio, y dadas las diferencias encontradas entre la forma de Nasa 1 y Nasa 5 y A. andegaviensis, hemos decidido clasificar por el momento el material estudiado en este artículo como Andegameryx aff. andegaviensis.

\section{Conclusiones}

Aunque el material es escaso, los dientes fósiles descritos en este trabajo permiten confirmar la presencia de Andegameryx Ginsburg, 1971 en el Mioceno inferior de las Bardenas Reales (Cuenca del Ebro), que ya había sido supuesta anteriormente a partir de restos postcraneales (Murelaga, 2000; 2004a). Si bien la especie de las Bardenas Reales es próxima a la especie tipo del género, Andegameryx andegaviensis, su talla es algo mayor que el material de esta especie procedente de localidades francesas, incluyendo la localidad tipo (Pontigné). Las dimensiones de las piezas estudiadas se sitúan en el rango de A. andegaviensis de Cetina de Aragón (Zaragoza, MN2). Morfológicamente, la forma de las Bardenas muestra algunas diferencias con $A$. andegaviensis, compartiendo ciertos rasgos con la forma clasificada como Andegameryx sp. de los yacimientos ramblienses de Loranca del Campo, Ramblar y Ágreda (Quiralte \& Morales, 2006; DeMiguel et al., en prensa).

El género Andegameryx está bien representado en el Ageniense-Rambliense (biozonas Y-A; Daams et al., 1987) de la Península Ibérica (cuencas del Duero, Ebro, Calatayud-Montalbán, Madrid y Depresión Intermedia). De hecho, el caso de la Península Ibérica presenta importantes particularidades en su fauna de rumiantes Pecora durante esta época. Así, de manera general, podemos decir que los géneros cervoideos inermes comunes en Centroeuropa (Amphitragulus, Dremotherium, Pomelomeryx, Bedenomeryx) pasan a ser minoritarios, o incluso estar totalmente ausentes, de la mayor parte de las asociaciones de rumiantes del Mioceno inferior de la Península Ibérica. Es en cambio Andegameryx el rumiante predominante en varias localidades, acompañado en ocasiones por formas afines como Teruelia y Lorancameryx.

\section{AGRADECIMIENTOS}

Este trabajo se ha llevado a cabo en el marco de los proyectos de investigación del Ministerio de Ciencia e Innovación y de los grupos de investigación CGL2007/66431/C02-02, CAMUCM 910607 y IT320-10 (Gobierno Vasco). Agradecemos a Alejandro Urmeneta (Comunidad de Bardenas Reales de Navarra) y a Eliseo Martínez (IES Valle del Ebro de Tudela) la ayuda que nos han proporcionado durante la realización del mismo.

\section{Referencias}

Alonso-Zarza, A.M.; Armenteros, A.; Braga, J.C.; Muñoz, A.; Pujalte, V.; Ramos, E.; Aguirre, J.; Alonso-Gavilán, G.; Arenas, C.; Baceta, J.I.; Carballeira, J.; Calvo, J.P.; Corrochano, A.; Fornós, J.J.; González, A.; Luzón, A.; Martín, J.M.; Pardo, G.; Payros, A.; Pérez, A.; Pomar, L.; Rodríguez, J.M. \& Villena, J. (2002). Tertiary. In: The Geology of Spain (Gibbons, W. \& Moreno, T., eds.). Geological Society, 293-334.

Daams, R.; Freudenthal, M. \& Alvarez Sierra, M. (1987). Ramblian: a new stage for continental deposits of early Miocene age. Geologie en Mijnbouw, 65: 297-308.

DeMiguel, D.; Quiralte, V.; Azanza, B.; Montoya, P. \& Morales, J. (in press). Dietary behaviour and competition for vegetal resources in two Early Miocene pecoran ruminants from Central Spain. Geodiversitas

Ginsburg, L. (1971). Un Ruminant nouveau des Faluns Miocènes de la Touraine et de l'Anjou. Bulletin du Museum National d'Histoire Naturelle, Paris, $2^{\circ}$ sér., 42: 996-1002.

Ginsburg, L. \& Morales, J. (1989). Les Ruminants du Miocene inferieur de Laugnac (Lot-et-Garonne). Bulletin du Museum National d'Histoire Naturelle, Paris., $4^{\circ}$ sér., 11: 201-231.

Ginsburg, L.; Morales, J. \& Soria, D. (1994). The Ruminants (Artiodactyla, Mammalia) from the Lower Miocene of Cetina de Aragón (Province of Zaragoza, Aragón, Spain). Proceedings of the Koninklijke Nederlandse Akademie Van Wetenschappen, 97: 141-181.

Larrasoaña, J.C.; Murelaga, X. \& Garcés, M. (2006). Magnetobiochronology of Lower Miocene (Ramblian) continental sediments from the Tudela Formation (wes- 
tern Ebro basin, Spain). Earth and Planetary Science Letters, 243: 409-423. doi:10.1016/j.epsl.2006.01.034

Linnaeus, C. (1758). Systema naturae per regna tria naturae, secundum classes, ordines, genera, species, cum characteribus, diferentiis, synonymis, locis. Tomus I. Editio decima reformata. Laurentii Salvii, Holmiae (Stockholm), Sweden, 824 pp.

Lourens, L.J.; Hilgen, F.J.; Shackleton, N.J.; Laskar, J. \& Wilson, D.S. (2004). The Neogene period. In: A Geologic Time Scale 2004 (Gradstein, F.N.; Ogg, J.G. \& Smith, A.G. eds.). Cambridge University Press, Cambridge, 409-440.

Morales, J.; Ginsburg, L. \& Soria, D. (1986). Los Bovoidea (Artiodactyla, Mammalia) del Mioceno inferior de España: Filogenia y Biogeografía. Paleontologia $i$ Evolució, 20: 259-265.

Morales, J.; Nieto, M.; Peláez-Campomanes, P.; Soria, D.; Álvarez, M.; Alcalá, L.; Amezua, L.; Azanza, B.; Cerdeño, E.; Daams, R.; Fraile, S.; Guillem, J.; Hoyos, M.; Merino, L.; de Miguel, I.; Monparler, R.; Montoya, P.; Pérez, B.; Salesa, M. \& Sánchez, I. (1999). Vertebrados continentales del Terciario de la Cuenca de Loranca (Provincia de Cuenca). In: La Huella del Pasado: Fósiles de Castilla-La Mancha. Patrimonio Histórico-Arqueología Castilla-La Mancha, Junta de Comunidades de Castilla-La Mancha, Servicio de Publicaciones, 235-260.

Murelaga, X. (2000). Estudio de las faunas de vertebrados del Mioceno inferior de las Bardenas Reales de Navarra y áreas colindantes (Cuenca del Ebro, Navarra). Tesis doctoral, Universidad del País Vasco/Euskal Herriko Unibertsitatea, Bilbao, 363 pp.

Murelaga, X.; Pereda-Suberbiola, X.; De Lapparent de Broin, F.; Rage, J.C.; Duffaud, S.; Astibia, H. \&
Badiola, A. (2002). Amphibians and reptiles from the Early Miocene of the Bardenas Reales of Navarre (Ebro Basin, Iberian Peninsula). Geobios, 35: 347-365. doi:10.1016/S0016-6995(02)00031-1

Murelaga, X.; Astibia, H.; Sesé, C.; Soria, D. \& PeredaSuberbiola, X. (2004a). Mamíferos del Mioceno inferior de las Bardenas Reales de Navarra (Cuenca del Ebro, Península Ibérica). Munibe, 55: 7-102.

Murelaga, X.; Larrasoaña, J.C. \& Garcés, M. (2004b). Nueva localidad fosilífera en el Mioceno inferior de las Bardenas Reales de Navarra (Cuenca del Ebro, Península Ibérica). Geogaceta, 36: 179-182.

Quiralte V. \& Morales J. (2006). Los Rumiantes (Artiodactyla, Mammalia) del Mioceno inferior de La Encinilla (Colmenar Viejo, Madrid). Estudios Geológicos, 62: 515-532. doi:10.3989/egeol.0662148

Scopoli, J.A. (1777). Introdvctio ad historiam natvralem sistens genera lapidvm, plantarvm, et animalivm hactenvs detecta, caracteribvs essentialibvs donata, in tribvs divisa, svbinde ad leges natvrae. Pragae, 506 pp.

Ruiz-Sánchez, F.J.; Murelaga, X.; Freudenthal, M.; Larrasoaña, J.C. \& Garcés, M. (in press a). Hypsodont Myomiminae (Gliridae, Rodentia) from the Lower Miocene Tudela Formation (Bardenas Reales, Ebro Basin, Spain) and their bearing on the age of the Agenian-Ramblian boundary. Geodiversitas.

Ruiz-Sánchez, F.J.; Murelaga, X.; Freudenthal, M.; Larrasoaña, J.C. \& Garcés, M. (in press b). A new Vasseuromys species from the Aragonian of Ebro basin (Spain). Acta Palaeontologica Polonica.

Recibido el 7 de marzo de 2011 Aceptado el 1 de septiembre de 2011 